\title{
Caroline Ziolko
}

\section{SARAH MOON, CONTE, PHOTOGRAPHIE ET VIDEO}

Réactiver le conte merveilleux par la vidéo créative

Quatre contes, d'après Hans Christian Andersen et Charles Perrault, écrits et illustrés par Sarah Moon, révèlent comment le photographique - photo et vidéo - et l'interrelation texte-image construisent un imaginaire fictionnel actualisé. Ces contes soulignent l'intérêt et les possibilités qu'offre une démarche plasticienne d'auteur pour transposer et réactiver les trames narratives de contes merveilleux plus que centenaires à partir de problématiques contemporaines.

RELIEF 4 (2), 2010 - ISSN: 1873-5045. P252-266

http://www.revue-relief.org

URN:NBN:NL:UI:10-1-101251

Igitur, Utrecht Publishing \& Archiving Services

(C) The author keeps the copyright of this article

\section{Réécrire le merveilleux}

Les technologies de l'image induisent de nouveaux langages visuels et de nouvelles approches créatives. Elles engendrent également un nouvel imaginaire via les représentations et mises en forme qu'elles permettent. C'est dans la réécriture de contes ou de récits anciens que ce phénomène est le plus évident.

Partant de ce constat on peut se demander: Quel est l'apport de la photographie et de l'image vidéo dans la mise en images de contes merveilleux ? En quoi l'imaginaire - défini par ces visuels - est-il actualisé ou distinct de celui construit par le dessin, la peinture ou la gravure? Comment la photographie plasticienne trouve-t-elle - entre l'objectivité mécanique du médium et la subjectivité du regard de l'auteur - sa place dans l'accompagnement de textes relevant d'une production orale ou d'une littérature européenne plus que centenaire? Les nouveaux médias alimentent aujourd'hui un imaginaire collectif, planétaire - plus vaste encore que celui couvert hier par l'imprimé - qui bien souvent s'origine dans une tradition lointaine. Si la photographie est principalement dédiée à la représentation du documentaire, du réel et de situations objectives, elle peut aussi, dans son interprétation plas- 
ticienne, parfaitement accompagner des récits fantastiques, merveilleux ou des situations surréalistes. A l'initiative de la Guilde du livre et des Éditions Clairefontaine, l'album photographique pour enfants a connu, à partir des années 1930, une certaine expansion. Après la Seconde Guerre mondiale, dans les années 1950, la photo accompagne la fiction et la narration et non plus seulement le récit documentaire. Sous l'impulsion du cinéma, l'actualisation de l'imaginaire merveilleux passe dès lors par le photographique - image argentique ou numérique, fixe ou animée et sonorisée. Ce dernier, entre rêve et réalité, devient alors un support narratif à part entière. D'autre part, réactiver un texte, un conte ou un récit relève d'une adaptation à un style d'écriture et à des problématiques contemporaines. Réécriture et nouveaux médias contribuent ainsi à l'actualisation de formes narratives anciennes et à l'élaboration d'un nouvel imaginaire collectif.

Ainsi, de la tradition orale au cinéma, ont été imaginées de multiples versions des récits merveilleux traditionnels qui pour bon nombre de scénaristes, sont une inépuisable source d'inspiration. Quelques trop rares créations d'auteur émergent par leur originalité d'une production de grande diffusion souvent insipide. Mais, parmi ces réussites, on peut retenir les récentes adaptations, par Sarah Moon, photographe et vidéaste de renommée internationale, d'œuvres de Hans Christian Andersen et de Charles Perrault. Ces créations d'auteur soulèvent différentes questions à propos de l'actualisation de l'image et du texte des contes anciens. Elles mènent aux interrogations suivantes: Quel est l'apport du photographique dans la mise en images de contes merveilleux, via l'imprimé ou la vidéo ? En quoi l'imaginaire généré par cette imagerie est-il actualisé, voire modifié, par une démarche, des outils et des supports de création et de diffusion contemporains ? Comment la photographie plasticienne trouve-t-elle ici sa place entre une imagerie mécanique et une représentation plus subjective?

Le photographique développe un langage visuel de plus en plus élaboré, capable de générer un contenu narratif ouvert et totalement polysémique. Au même titre que l'imprimé, le DVD est un support de diffusion des contes, légendes et autres fictions où le texte - en voix off - et l'image se font écho. Questionné aujourd'hui, le rapport texteimage ne peut donc pas faire l'économie d'une interrogation sur les outils, les supports et les moyens de représentation ou de diffusion. Ces interrogations surgissent en amont - ou en parallèle - d'une analyse du style de l'auteur et du contenu sémantique des productions. Par rapport 
à ces interrogations, les productions de Sarah Moon sont des cas d'étude très pertinents.

\section{Du prêt-à-porter au conte merveilleux}

Après une carrière aussi et originale que créative dans la photographie de mode, Sarah Moon, née en 1927, s'intéresse, à partir des années 1985, à la mise en images et à la réécriture de contes merveilleux européens du XVII et XIXe siècle. A travers une approche multimédia originale, ses productions transportent le public dans un imaginaire onirique quelque peu décalé et poétique, abordant des thèmes intemporels et des problématiques en phase avec le quotidien, la vie en société ou l'actualité médiatique contemporaine. Dans une série d'albums - édités au Japon en 2003, 2005, 2006 et 2007, par le Kahitsukan, Kyoto Museum of Contemporary Art -, Sarah Moon réactive quatre contes de Charles Perrault et de Hans Christian Andersen.

Chaque album est accompagné d'un DVD. Le texte alterne avec une photographie ou une séquence de clichés. Photographie, texte et vidéo offrent ainsi une lecture multimodale et complémentaire d'un même conte. Bien que ces quatre productions ciblent un public de 11 à 16 ans, elles peuvent aussi intéresser un auditoire beaucoup plus large, par leur esthétique sophistiquée - celle de la photographie plasticienne contemporaine - et par les différents niveaux de lecture qu' offre chaque texte. A Paris, le CRDP propose une compilation de ces quatre contes pour une exploitation pédagogique accompagnée - un petit livret propose des pistes d'exploitation pédagogique par niveau de scolarisation -, facilitant ainsi la relation entre la réception de ces œuvres dans le cadre d'un programme scolaire précis.

L'analyse des formes et contenus de ces documents part ici d'une approche visuelle et sémiologique. L'approche visuelle permet de décrire et d'interpréter chaque page, double-page, image fixe et plan vidéo. Adopter la sémiologie des langages visuels - photographie, imprimé, vidéo - comme point de départ et repère méthodologique permet d'analyser le rapport texte-image selon une approche vérifiable, comparative et transposable d'un ouvrage à l'autre pour définir la démarche de l'artiste. Cette approche a, d'autre part, l'avantage d'établir les bases d'une démarche transdisciplinaire accessible et logique.

L'exploration des visuels procède selon trois étapes. Ciblant en premier les données repérables lors d'une lecture purement optique, 
elle identifie les variables visuelles - de forme, de taille, de place, de valeur et de couleur, de grain. La plasticité du photographique peut ainsi être définie selon des critères précis et comparables. Elle identifie ensuite les composantes figuratives - c'est-à-dire les items - que l'on peut nommer précisément et retracer au fil des séquences visuelles. Le contenu narratif, voire documentaire, peut ainsi être précisé. Enfin, elle analyse les connotations induites - autant sur l'image fixe qu'en vidéo. A ce stade on peut repérer les arguments visuels de l'imaginaire contemporain en termes de connotations et de niveaux de lecture de l'image, seule ou par rapport au texte.

L'analyse du langage-image vidéo doit, par ailleurs, faire l'objet d'une approche spécifique - bruitage, voix off, structuration des séquences sonores et animées. Enfin, ces observations permettent d'observer parallèlement la structure narrative de l'imprimé et de la vidéo d'un même conte ou de plusieurs contes différents. Les visuels figurant dans ces albums sont tirés des séquences vidéo ; et les textes reprennent les paroles - écrites et lues par Sarah Moon elle-même - que l'on entend en voix off dans les vidéos.

L'analyse de la plasticité des productions s'intéresse au cadrage, à la composition, au traitement de la lumière, selon une approche que nous avons spécifiquement mise au point, dès 1973, pour décrire l'image photographique.

\section{Photographie et narration : $d u$ réel à l'imaginaire}

Dans Souvenirs improbables Sarah Moon (Sarah Moon et Robert Delpire éditeur, imprimé en Suisse, 1981), Danièle Sallenave précise en avantpropos: «Voici des photographies de commande... des photographies où la beauté elle-même est une beauté asservie : mise au service d'une marque, d'un objet, d'un produit. » En effet, le traitement que Sarah Moon applique et même inflige à ses clichés - agression de la matérialité du négatif, intervention au développement et au tirage en laboratoire - est très tôt devenu la marque distinctive d'une pratique photographique contemporaine. Une pratique qui revendique l'usage du médium comme outil et support d'une expression plasticienne à part entière ; et qui, partant de prises de vue en studio ou en extérieur, induit - via la plastique de l'image - une dérive narrative vers l'imaginaire, le rêve et la poésie visuelle. "Je m'évade dans la photographie. Je pars d'ici pour ailleurs, le monde dans mes bagages, je ne sais jamais pour quelle destination, je sais seulement que là-bas, le soleil se couche avant de se le- 
ver. » (Altieri et Delpire 2003, 65) Interrogée sur ses choix thématiques, à savoir pourquoi elle n'avait pas retenu Cendrillon ou La Belle au bois dormant, vu son expérience de la publicité, la photographe répondait à Janine Kotwica : "Ce que j'aime dans les contes n'a rien à voir avec l'époque où ils se situent. Je crois au contraire que c'est leur symbolique intemporelle qui m'intéresse et même si je devais raconter ceux que vous citez, je leur enlèverais leur décorum pour mieux les "récupérer" ». L'exposition organisée à l'Hôtel des Arts de Toulon, en 2003, restituait parfaitement le concept de cette esthétique photographique. Dans l'œuvre plasticienne de Sarah Moon, sans le moindre texte, chaque cliché fait récit. Chaque image, à travers une plastique souvent complexe de clairs-obscurs, de cadrages forts et dépouillés et d'effets visuels monochromes propres au photographique, ouvre de multiples pistes d'interprétation. Le catalogue de l'exposition de Toulon rassemble en vrac des thématiques simples et conventionnelles. La frêle silhouette d'une femme traverse un jardin entre l'ombre d'une maison et celle d'un arbre - Ombres portées, 1992. Le crâne d'une mouette en plein vol apparait, dans le ciel, comme une tête de mort - La mouette, 1998. Un éléphant est assis au milieu d'une piste de cirque - L'éléphant, 1999. Deux rhinocéros, cadrés sur fond de colline - Histoire naturelle, 1995. Le profil noir de trois palmiers s'inscrit sur les façades aveugles d'immeubles surmontées d'antennes de télévisions - Sao Paulo, 2000. Au dessus d'un bâtiment, une étoile filante constituée d'ampoules électriques n'est plus qu'un angoissant graphisme monochrome - Knocke le Zoute, 1990. Un panneau directionnel lumineux est survolé par trois hirondelles - Exit, 1998. Le drapé marmoréen d'un buste classique évoque, via le traitement de la pellicule, un dessin au fusain - La robe de l'ange, 1999. Certaines de ces images apparaitront ici où là dans un conte. L'observateur de toutes manières retrouvera dans les contes l'ambiance flottante et angoissante de ces clichés monochromes. Un style qui contient nostalgie et sérénité. Et qui parle autant à la mémoire qu'à l'imagination du public. Au niveau de la présentation des clichés, le cadre de l'image laisse apparaitre les bords du négatif. Traduction graphique directe d'une certaine technologie - celle du film argentique et du polaroïd; du respect, lors du tirage sur papier, du cadrage initial lors de la prise de vue. Mais ce détail infime identifie aussi une démarche plasticienne qui revendique sa filiation au registre des arts visuels et même aux beaux-arts. En effet, la présence de ce cadre graphique, aléatoire et historiquement daté, délimite et authentifie le statut artistique et créatif de l'œuvre. La photogra- 
phe affirme même, dans Souvenirs improbables Sarah Moon, à Danièle Sallenave qui l'interroge alors sur son rapport à la réalité :

[...] oui je la détourne à mon profit, je la fais mienne. [...] je l'arrache à son contexte, je lui donne une autre vie, un autre sens, une autre histoire, à la fois celle de notre rencontre à cet instant-là et celle du souvenir qu'il réveille et qui n'a probablement rien à voir avec lui, mais dont il devient le symbole. (Moon et Delpire, 1981, 63)

De même que le conteur personnalise chacune de ses prestations par des inflexions de la voix, par des gestes ou des arguments narratifs de son cru, la photographe identifie ses créations par divers arguments plastiques distinctifs. Il est intéressant de noter que ce traitement graphique distinctif du cadre se retrouve aussi sur certains clichés de prêtà-porter produits par la photographe. A propos de ces créations plus fonctionnelles, Altieri évoque les Fragments d'un monde flottant (Altieri et Delpire 2003, 5-7) et l'ambigüité que dégage la plastique photographique chez Sarah Moon. Cette plasticité n'est pas complaisante, elle interroge et perturbe même le public.

Les images à la beauté rêveuse et mélancolique se transmuent en visions inquiétantes et oppressantes, surgissement d'un monde clos et anaérobique dans lequel le temps s'est figé comme sous l'emprise d'un sommeil narcotique ; le doute et l'incertitude s'installent alors dans l'esprit du visiteur qui est insidieusement conduit à remettre en cause la réalité de ce qui est offert à sa vue. (Moon 2003, 5-7)

Évoquant l'historique du légendaire conflit entre l'Art pour l'Art et la réponse à la commande, Danièle Sallenave, pour sa part, conclut en situant, entre fiction et théâtralité, un regard d'auteur qui, partant de l'imaginaire du prêt-à-porter, juxtapose des séries de micro récits où les corps et les personnages nous invitent à interroger le sens même de l'existence humaine. Or, c'est précisément ce que proposent métaphoriquement les contes et récits populaires. Secteur que va peu à peu explorer l'artiste-photographe. Retenant, en fonction de sa sensibilité, les contes les plus connus et les plus propices à l'expression d'une dérive très contemporaine vers l'imaginaire, le rêve et la poésie visuelle.

Le traitement pictorialiste confère aux clichés de mode de Sarah Moon une originalité poétique et une irréalité qui tranche avec leur finalité fonctionnelle. Mais ce traitement convient parfaitement à ses pro- 
ductions d'auteur. D'ailleurs certaines photographies - créés dans le cadre de commandes de mode - préfigurent les illustrations photographiques ou des cadrages vidéo qui, deux décennies plus tard, accompagneront les contes imaginés par la photographe.

\section{Un imaginaire merveilleux décalé et actualisé}

Cette maîtrise de l'ensemble des paramètres narratifs inclut la reformulation, dans un langage contemporain, des actions-clés des contes les plus connus du répertoire européen ancien. Ceux que chacun d'entre nous a entendus dans son enfance. Cette adaptation personnelle des scénarii fait éventuellement écho à l'actualité médiatique la plus banale. On découvre ainsi que le légendaire Barbe Bleue est, aujourd'hui, un sérial killer, devenu imprésario. Il épouse, puis assassine ses victimes après leur avoir fait miroiter une carrière prometteuse. Pour avoir croisé la désillusion dans le regard des modèles sur les podiums des défilés de mode, la photographe sait trouver les images justes pour traduire, sans pathos ni mièvrerie aucune, cette dérive métaphorique dont certaines, à l'instar de son héroïne, réchappent pour retrouver la lumière et la vie loin de toute fausse représentation. En arrière plan de ses contes, Sarah Moon choisit d'évoquer le fait divers, l'histoire locale. Sur le ton du souvenir et de la mémoire, la conteuse évoque l'histoire de personnes connues ou rencontrées jadis. Cette identification ancre le récit dans l'ambiance d'une époque, d'une ville, d'une rue, d'une demeure... Elle lui confère plus de crédibilité. Enfin, en voix off, le débit monocorde de la narratrice ajoute une note de réalisme à ces histoires somme toute banales. Comme celles que l'on peut lire chaque matin, à la une des journaux. Certains faits divers tournent mal. C'est la vie. Ils deviennent alors des histoires que l'on évoque d'une voix un peu lasse.

Ces adaptations évoquent, de près ou de loin, les problèmes de la société contemporaine; et concernent par conséquent un public jeune. Elles abordent, par exemple, le problème des greffes d'organes réalisées dans des cliniques parallèles, opérant dans les conditions les plus glauques. C'est la thématique soulevée dans La Sirène d'Auderville, ( $\mathrm{d}$ 'après La Petite Sirène, de Hans Christian Andersen). Mais, ici en arrière plan, il est également question de la quête désespérée d'un changement de statut physique. Le conte imaginé, par Sarah Moon, laisse deviner que l'opération entreprise, par la petite sirène, doit lui donner accès à un autre cadre de vie. Mais ce qui motive principalement sa démarche, c'est l'espoir de retrouver sur terre un scaphandrier qu'elle a sauvé de la 
mort. A la suite de l'opération qui doit lui permettre de marcher, la sirène échoue, au propre comme au figuré, sans voix, sur une plage normande près d'un bunker. Là où elle trouvera également la mort.

La vente d'une maison familiale et la destruction des souvenirs se dessinent en filigrane dans L'Effraie - c'est l'adaptation vidéo du Vaillant soldat de plomb, conte initialement imaginé par l'écrivain danois ; histoire que complique, sur fond de bombardement, une relation affective malheureuse entre deux enfants.

Circus n'aborde pas seulement la dérive d'un petit cirque et celle de ses saltimbanques, tout aussi gris et effacés par la monotonie de leur numéro que par le grain de l'image. Circus, c'est aussi la dérive affective d'une fillette, abandonnée par sa mère. Cette écuyère a choisi de quitter sa famille et le cirque où elle se produit tous les soirs avec un lanceur de couteaux d'une improbable dextérité. Cette Petite marchande d'allumettes est une enfant des années 50. L'éclairage des rues, l'équipement rudimentaire du cirque et cette petite communauté de déshérités devient un décor générique. Celui d'une époque et d'un style de vie nomade, celui des gens du voyage qui tournent en rond comme les animaux de leur ménagerie. Mais, c'est aussi un voyage à la dérive vers la mort, le froid et l'oubli. Celui d'une enfant seule dans une ville, le soir même du jour de l'an, au milieu de l'indifférence des passants et des voitures qui l'éclaboussent.

D'autres thématiques, plus génériques, hantent les récits de Sarah Moon, mais le tragique et la mort en font toujours partie. Un seul des quatre contes, Le fil rouge, trouve une heureuse conclusion. Échappant à un serial killer, alias un faux imprésario qu'elle vient d'épouser sans conviction, l'innocente apprentie chanteuse réussit à s'échapper de la demeure de son bourreau. Non sans avoir découvert les portraits de celles qui l'ont précédée dans cet appartement - mi loft d'artiste, mi usine désaffectée - où son époux l'a enfermée. Le château de Barbe bleue est ainsi reformulé dans un décor périurbain générique en demi-teintes grises que seul vient animer le fil rouge de l'intrigue. Les photographies des victimes, découvertes par la jeune-fille, se substituent à tout argumentaire. La disposition des images - sous la forme d'une installation introduit dans cet espace narratif l'œuvre de Christian Boltanski, un plasticien qui travaille sur la mémoire et l'image de personnes disparues. Ici encore, l'image fait sens et récit à plusieurs niveaux, sans qu'il soit indispensable de disposer de références artistiques précises. La présence d'une œuvre d'art introduit, à ce niveau, une connotation tempo- 
relle et mémorielle supplémentaire. Une parenthèse ouverte sur une interprétation parallèle de la mort, de la détention, de l'arbitraire : thématiques retenues par l'artiste. C'est donc autant l'imaginaire d'adolescents d'aujourd'hui que l'imaginaire de la réalisatrice qui trouvent ici un écho direct dans le portrait de cette jeune chanteuse et dans les situations et le décor de cette vidéo. Mais ici, plus que dans les autres contes, le réalisme, voire le naturalisme de situation, évacue tout propos visuel ou littéraire apparenté au conte merveilleux. Selon une conclusion du type Happy End, loin de tout pathos, l'héroïne réussit à s'échapper. Elle est devenue moins naïve et elle reprend son destin en main. Sous une forme différente, le public retrouve une des thématiques de Hans Christian Andersen. Ici, au delà de l'anecdote, l'ex-photographe de mode dépeint le portrait d'une jeune fille de notre époque qui sacrifie certains de ses rêves d'adolescente - le souvenir de son premier amour - à ses ambitions professionnelles de chanteuse. En réalité, c'est le mariage et l'enfermement dans le domicile conjugal qu'elle gagne à ce petit troc. Se rendant compte alors de ce qui métaphoriquement l'attend si elle reste auprès de son mari, elle quitte en courant cette forteresse d'ombres et de souvenirs pour courir tout droit devant elle en pleine lumière.

\section{Images et connotations photographiques}

Le style photographique développé par Sarah Moon induit des connotations spécifiques qui font sens et alimentent parfaitement ces propos fictionnels qui se situent entre rêve et réalité. Les jeux de lumière sont ici essentiels. Ils tissent une trame de connotations complémentaires qui se font écho et conduisent le récepteur sur les pistes d'une lecture métaphorique. Or, c'est le réalisme même du récit et du traitement de l'image qui induisent ces différentes métaphores. L'usage, dans Le fil rouge, d'une très ponctuelle touche monochrome rouge, dans le film en noir et blanc, dramatise et rehausse l'action et le décor. Les scènes où le surréalisme - et non le merveilleux - intervient font référence ici à l'imaginaire de la jeune fille. Ces séquences oniriques traduisent, à l'écran, le décalage que la jeune fille perçoit entre le réel, où elle évolue, et ses attentes, ses aspirations et les promesses de son pseudo imprésario. Et là, le sordide de la situation rejoint le rêve, ou plutôt le cauchemar. Autre image surprenante, lors du mariage avec l'imprésario, une oie, une fourchette plantée dans le dos, s'envole et traverse la salle du banquet.

Les objets, mais aussi l'animation rendue possible en vidéo véhiculent non seulement un imaginaire d'un style particulier, très crédible, 
mais aussi des connotations plus diffuses relevant de la culture et du parcours de l'auteur. C'est par exemple la présence surréaliste, de ce bouquet de mariée en fer forgé, à l'image des ornementations déposées autrefois sur les pierres tombales, qui est offert à la jeune.

Le traitement plasticien de l'image fixe ou animée engendre chez Sarah Moon une dynamique particulière. Stimulé par cette succession d'images plurielles, le récepteur doit remettre constamment en question sa propre lecture de l'image. C'est une démarche totalement distincte de celle proposée par le conte traditionnel, où des redondances de mots, d'images mentales et de déclinaisons narratives souvent très convenues finissent par faire obstacle à toute vigilance, à tout sens critique et à toute remise en question de la forme et du contenu délivrés. Les contes d'autrefois étaient bien souvent utilisés, le soir, pour endormir les enfants. Ceux de Sarah Moon éveillent au contraire la curiosité et l'esprit critique du public via la richesse narrative de la photographie ou de la vidéo.

Cette relecture de contes européens anciens, orchestrée par Sarah Moon, conserve la structure narrative et les clés des contes populaires tels qu'ils ont été définies par Vladimir Propp. C'est-à-dire, selon des séquences d'actions qui construisent des profils types de contes. La créativité de la narratrice consiste, ici, à reformuler et actualiser les situations ; à inventer des portraits, des actions et des contextes totalement actuels et à créer de nouvelles fictions où les visuels s'apparentent à des archives des années 1940.

Le récepteur n'a pas à choisir ici entre deux logiques, deux univers, deux conceptions du monde. Il n'a pas à opter pour la rationalité contre le merveilleux ni l'inverse. L'image est là - en contrepoint - à chaque instant, non pas pour suppléer à son imaginaire défaillant ou restrictif. Bien au contraire, l'image accompagne et développe la part cachée - ou sous entendue - du texte. Quand le récit s'oriente vers plus de réalisme, la vidéo plasticienne rétablit l'équilibre en faveur d'une poésie multidimensionnelle - visuelle, sonore, narrative. Quand la vidéo affiche des images d'esprit surréaliste, le propos - en voix off - rétablit une certaine objectivité, non pas descriptive mais narrative. Entre rationalisme et symbolisme, imaginaire et réalité, Sarah Moon, auteur, récitante et vidéaste tisse une fiction poétique et sensible dont les racines multiples trouvent un écho profond dans notre imaginaire collectif. Cependant, ces récits revendiquent aussi clairement leurs racines littéraires anciennes. La jaquette du DVD proposé par le CRDP et le livret 
d'accompagnement situent très clairement - en vue d'une exploitation pédagogique - l'articulation narrative et les sources de chaque film.

\section{L'univers mythique imaginé par Sarah Moon}

Sarah Moon ancre très précisément ses récits dans la vie et la culture contemporaine. Elle prend ainsi quelques distances par rapport au conte merveilleux et de ce fait inscrit son propos dans l'univers du mythe :

en effet, le merveilleux, dans le conte, déjà très conventionnel, se trouve coupé d'une ethnographie concrète, de croyances et de prescriptions rituelles, qui, elles, sont nettement délimitées par chaque culture (aussi bien sur le plan ethnique que sur le plan historique). (Propp, 190-219)

Mais, les croyances et rituels auxquels sont soumis les personnages ne sont ici autres que ceux de notre quotidienneté - directement vécue ou perçue à travers la presse et les médias. Dans chaque conte, l'inscription de l'action dans l'Historique est évidente, tant par les diverses connotations induites par le style des costumes des personnages que par la mise en images du récit.

Si le photographique fait référence à une éventuelle authenticité des faits, la morphologie du conte articule les actions et la temporalité du récit en transposant la logique narrative dans l'univers du souvenir et de la fiction. La polysémie de l'image trouve ainsi, dans la structuration simplifiée du récit, un équilibre judicieux. Aussi, l'auteur prend ses distances avec un propos merveilleux classique et s'oriente vers une formulation littéraire contemporaine, tout en conservant, comme c'est le cas pour les récits les plus anciens, une dimension symbolique.

L'univers narratif, imaginé par Sarah Moon, est un monde clos sur lui-même. Bien que l'intrigue, par épisode, fasse intervenir quelques paysages extérieurs, comme une campagne avec des arbres et des plages nordiques - L'Effraie, La Sirène d'Auderville; ou des rues, des voies ferrées et des paysages urbains lugubres - Circus, Le Fil rouge. Mais, ces décors se combinent par couples antagonistes : l'intérieur / l'extérieur ; la maison / l'ailleurs; le cirque / la voie ferrée ; le fond de l'océan / le blockhaus ou la plage... Le récit évoque, d'autre part, le parcours des personnages entre : l'enfance / l'âge adulte ; la vie / la mort ; le Bien / le Mal... A travers les plans de L'Effraie, on découvre une demeure familiale abandonnée, versus un paysage champêtre et un hangar à foin dé- 
vasté par la guerre. Dans l'univers africanisant et morbide d'un taxidermiste, une idylle enfantine va évoluer entre guerre et paix pour trouver une fin fictive tragique. Mais la vie reprendra ses droits avec l'arrivée de jeunes propriétaires. Des portraits du début du siècle s'opposent ainsi aux cadrages de vrais enfants jouant à être des images ou des soldats de plomb. Dans Le fil rouge, l'Ailleurs, c'est la cour de l'immeuble et ses banquets cauchemardesques sans convives; la fête foraine et ses monstres grimaçants. L'enfermement des tristes locaux d'audition ne s'ouvre que sur un appartement-prison sombre où s'entassent les cadavres d'un serial killer. Ici, seule une énergie désespérée permettra à la jeune chanteuse trop crédule de fuir cette intériorité morbide pour s'évader au dehors vers la lumière afin de rompre ce cycle infernal où elle s'est laissée entraîner malgré elle.

La mise en images articule aussi diverses paires sémiotiques complémentaires ou opposées : le typographique / le photographique ; le monochrome / la couleur ; l'image fixe / le montage vidéo ; la voix off / les bruitages. La voix off très monocorde de la conteuse s'oppose en effet à des sons et des bruitages plus incisifs. Les jeux d'ombre et de lumière jouent également un rôle essentiel. Dans ces films en noir et blanc, une très ponctuelle trace de couleur vive focalise l'attention. Le fil rouge trace le titre. Il devient ensuite couleur dominante sur quelques plans séquences - une fête foraine, la salle où sont enfermés les restes des victimes du faux imprésario.

Le symbolique, chez Sarah Moon fait davantage appel, dans le propos visuel ou littéraire, aux mythes des cultures anciennes tels qu'ils ont été étudiés par Claude Lévi-Strauss, qu'aux contes populaires dont l'articulation a été formulée par Vladimir Propp. La constante thématique première concerne, ici, le lien affectif, décliné par âge, genre, contexte, période. De l'amour filial désintéressé à la passion destructrice ou contrariée, les situations psychologiques sont sans issue ou très limitées. Comme dans les contes de Hans Christian Andersen, le parcours des personnages est tracé d'avance, par l'Autre ou par le destin. Telle est la toile de fond de cet univers fictionnel qui, une fois le livre fermé, disparaît pour faire place à la réalité. Le lecteur étant dès lors averti est capable de prendre en main sa destinée et de faire ses propres choix. Entre fiction et réalité, tout s'articule dès lors en binômes signifiants.

Dans Circus, l'amour fatal d'une acrobate pour un étranger la conduit à quitter sa fille et sa communauté. Ce qui plonge le petit cirque où elle se produit dans la misère et oblige la fillette abandonnée à tenter 
de vendre des allumettes dans la rue pour survivre, avant qu'elle ne meure de froid, la nuit du Réveillon, sous le regard des passants indifférents. Son amour filial inconditionnel ne lui apporte pour tout réconfort que quelques visions fantastiques de sa mère en costume de scène. Ce cirque, ses artistes et sa dérisoire ménagerie constituent un monde clos, communautaire et solidaire. A l'opposé, la rue, ses voitures et ses passants indifférents ne sont synonymes que de froideur, de faim et de mort pour cette fillette partie chercher de quoi survivre.

L'amour innocent de deux enfants - L'Effraie - sera brisé comme le hangar où ils se retrouvent. Entre la campagne ravagée par la guerre et la propriété familiale "d'un taxidermiste venu de Lituanie au début $\mathrm{du}$ siècle dernier, qui à sa mort avait laissé une collection d'animaux empaillés ", le drame se joue dans un huis clos campagnard mais bourgeois. «Le soleil ne se couchait plus. La guerre était partout». D'un plan à l'autre, l'image monochrome confond photos anciennes, statuettes et personnages réels.

Le rapport du conte à la psychanalyse se profile en filigrane dans le caractère onirique de certaines images. Dans certains cas, l'articulation du visuel fait place à l'expression, aussi suggestive que poétique, de phantasmes inconscients que l'enfant comme l'adulte, peuvent investir de leur propre lecture. Dans Psychanalyse des contes de fées, Bruno Bettelheim, en 1976, s'est intéressé au rapport étroit qui s'instaure entre le récit merveilleux et la psychanalyse. Mais l'ouvrage n'aborde pas la dimension visuelle des illustrations ; encore moins celle du photographique. Or, l'esthétique de l'image est indissociable de sa dimension symbolique. Elle dégage et oriente de très complexes pistes d'interprétation. Cette lecture plurielle sollicite continuellement l'inconscient et stimule l'imaginaire individuel ou collectif du récepteur, de manière plus dynamique que les propositions émanant des formulations anciennes du conte merveilleux. Et la qualité polysémique de l'image conduit l'imaginaire du récepteur bien au-delà du texte.

Le merveilleux s'est aujourd'hui laïcisé et correspond aux grandes interrogations de la société contemporaine. Mais, chez Sarah Moon, il conserve une dimension surréaliste, intemporelle et magique. La magie aujourd'hui ne réside donc plus seulement dans l'action décrite par le conte, mais dans une mise en images syncrétique. Le public se trouvant ainsi un instant immergé via la photographie ou la vidéo, dans un univers fictionnel à mi chemin entre le réel et l'imaginaire. Le photographique offre d'autre part une alternative par rapport à des technolo- 
gies plus récentes de simulation du réel qui, en impliquant d'avantage le corps et l'esprit dans une fiction pré formatée, laissent peu de place à la réflexion, à l'appréciation des images, et par conséquent à la distanciation et au sens critique vis-à-vis des récits proposés ou même tout simplement au rêve.

Si le travail de la photographe s'est longtemps inscrit dans le cadre d'une production médiatique grand public et commerciale - celui de la photographie de mode - elle a toujours cherché à dire, au delà de l'image, ce qui l'intéressait derrière l'image: la narration. Elle raconte une histoire en une, deux ou plusieurs images fixes, monochromes ou non. L'histoire, réelle ou fictive, du modèle photographié. Si les contes expliquent au quotidien, depuis la nuit des temps, la vie, l'amour et la mort, les photographies de Sarah Moon ont, semble-t-il, toujours tenté de traduire des histoires intimes, réelles ou imaginaires, mais aussi collectives. Elle ne s'est donc pas improvisée conteuse. Elle n'a fait que transposer, avec la vidéo et le conte une démarche personnelle, un regard, un savoir-faire et un imaginaire créatif à l'écoute de son temps, qui sait aussi témoigner de manière métaphorique de la mémoire de lieux, des gens, des images et de la pérennité d'une intemporelle tradition narrative.

\section{Ouvrages cités}

Bruno Bettelheim, Psychanalyse des contes de fées, Pari, Robert Laffont, 1976.

Janine Kotwica, «Sarah Moon, Le vacillement du temps et des lumières », Parole 3/09, (2008).

Sarah Moon, Textes de Robert Delpire et Sarah Moon, Paris, Nathan, Photo Poche, 1998.

Sarah Moon, Textes de Gilles Altieri et Robert Delpire, Entretien avec Sarah Moon recueillis par Magali Jauffret, Hôtel des Arts - Centre Méditerranéen d'Art - Conseil général du Var, Toulon, 4 octobre /16 novembre 2003.

Sarah Moon, Circus, Texte, film et photographies de Sarah Moon, Kahitsukan, Kyoto Museum of Contemporary Art, 2003.

—, L'Effraie, Un film de Sarah Moon, Texte, film et photographies de Sarah Moon, Kahitsukan, Kyoto Museum of Contemporary Art, 2004.

- Le Fil rouge, The Red Tread, Texte, film et photographies de Sarah Moon, Kahitsukan, Kyoto Museum of Contemporary Art, 2005.

—, La Sirène d'Auderville, The Mermaid of Auderville, Un film de Sarah Moon, Texte, film et photographies de Sarah Moon, Kahitsukan, Kyoto Museum of Contemporary Art, 2007. 
Souvenirs improbables Sarah Moon, Interview de Danièle Sallenave, Sarah Moon et Robert Delpire éditeur, imprimé en Suisse, 1981.

Vladimir Propp, Morphologie du conte, Paris, Seuil, 1965 et 1970. 